\title{
Distribution of antibiotic-resistant bacteria in aerobic composting of swine manure with different antibiotics
}

\author{
Tingting Song ${ }^{1,2}$, Hongna Li ${ }^{1}$, Binxu Li ${ }^{1}$, Jiaxun Yang ${ }^{1}$, Muhammad Fahad Sardar ${ }^{1}$, Mengmeng Yan ${ }^{1}$, Luyao Li ${ }^{1}$, \\ Yunlong Tian', Sha Xue ${ }^{2,3^{*}}$ and Changxiong Zhu ${ }^{1 *}$ (D)
}

\begin{abstract}
Background: Livestock manure is an important reservoir of antibiotic-resistant bacteria (ARB) and antibioticresistance genes (ARGs). The bacterial community structure and diversity are usually studied using high-throughput sequencing that cannot provide direct evidence for ARB changes. Thus, little is known about the distribution of ARB, especially in the presence of different antibiotics in composting process. In this study, the fate of ARB was investigated in aerobic composting of swine manure, using chlortetracycline, sulfamethoxazole, lincomycin, and ciprofloxacin as typical antibiotics. The abundance and species of ARB were analyzed systematically to evaluate their ecological risk at different stages of composting.
\end{abstract}

Results: The absolute abundance of total ARB decreased, while the relative abundance increased on day 2 . The relative abundance of lincomycin-resistant bacteria was higher than other ARBs during the whole composting process. The absolute abundance of four ARBs was $9.42 \times 10^{6}-2.51 \times 10^{2} \mathrm{CFU} / \mathrm{g}$ (lincomycin- > chlortetracycline- > sulfamethoxazole- > ciprofloxacin- > multiple antibiotic-resistant bacteria), and they were not completely inactivated at the end of composting. Antibiotics led to a partial proliferation of ARBs including Corynebacterium, Sporosarcina, Solibacillus, and Acinetobacter. Especially, Corynebacterium, a pathogenic genus, was observed in chlortetracycline and lincomycin treatments.

Conclusion: Among the antibiotics studied, lincomycin showed the highest ecological risk, due to it expanded the range of lincomycin-resistant bacteria at the phyla level (Firmicutes, Actinobacteria, and Proteobacteria). The principal co-ordinates analysis indicated that the bacterial community structure was primarily associated with the composting stages rather than antibiotic types. Possible potential hosts and the related to the decrease of ARGs abundance were indicated based on the network analysis. The decrease of culturable Proteobacteria and the increase of culturable Firmicutes (Solibacillus, Bacillus) partially explained the high degradation rate of various ARGs with the progress of composting in this study. These results provided important information for the control of antibiotic resistance in composting.

Keywords: Antibiotic-resistant bacteria, Lincomycin, Aerobic composting, Ecological risk

\footnotetext{
*Correspondence: xuesha100@163.com; zhuchangxiong@caas.com ${ }^{1}$ Institute of Environment and Sustainable Development in Agriculture, Chinese Academy of Agricultural Sciences, Beijing 100081, People's Republic of China

${ }^{2}$ College of Forestry, Northwest A\&F University, Yangling, Shaanxi 712100,

People's Republic of China

Full list of author information is available at the end of the article
}

\section{Background}

Antibiotics have been widely utilized in animal husbandry, and their residual concentrations were reported in the $\mu \mathrm{g} / \mathrm{kg} \sim \mathrm{mg} / \mathrm{kg}$ level in livestock manure [1-3]. In addition, inadequate assimilation in the animal body has led to increase residual antibiotics in the manure, which
SpringerOpen

(c) The Author(s) 2021. Open Access This article is licensed under a Creative Commons Attribution 4.0 International License, which permits use, sharing, adaptation, distribution and reproduction in any medium or format, as long as you give appropriate credit to the original author(s) and the source, provide a link to the Creative Commons licence, and indicate if changes were made. The images or other third party material in this article are included in the article's Creative Commons licence, unless indicated otherwise in a credit line to the material. If material is not included in the article's Creative Commons licence and your intended use is not permitted by statutory regulation or exceeds the permitted use, you will need to obtain permission directly from the copyright holder. To view a copy of this licence, visit http://creativecommons.org/licenses/by/4.0/. 
could promote selection for antibiotic-resistant bacteria (ARB) and antibiotic-resistance genes (ARGs) and making them a major reservoir of antibiotic resistance $[4,5]$. In recent decades, several techniques have been adopted to determine the variations of microbial structure. Traditional microbiological methods are essential and indispensable as a means to culture, and have continuously been used to successfully isolate various microbes [6]. The survival ability of bacteria in the presence of antibiotics is the only way to evaluate whether they are antibiotic-resistant or not, with the minimum inhibitory concentration quantified by agar dilution or broth microdilution assays [7]. Salmonella and Campylobacter, pathogenic genera, have been detected at $10^{5}$ colony-forming units $(\mathrm{CFU} / \mathrm{mL})$ and most of which were antibiotic resistance in the pig farm lagoons [8]. Numerous antibioticresistant human pathogenic genera, Escherichia coli, Alcaligenes faecalis, and Enterococcus, have been found in chicken manure [9]. Extensive use of antibiotics in animal husbandry significantly increased ARB and ARGs in livestock settings [10]. This would lead to deleterious implications on human health and environmental quality [5]. Consequently, it is particularly important to reduce the spread and diffusion of ARB and ARGs.

Microorganisms are essential for composting, and the compositions of these microbial communities are altered during this process. For example, lincomycin (LIN) residues promoted the abundance of Firmicutes, Actinobacteria, Proteobacteria, Chloroflexi, and Bacteroidetes in composting [2]. Previous studies found that the proportion of Chloroflexi was higher than other phyla, and the composting process also resulted in increases of Bacteroidetes abundance. The increase paralleled the antibiotic concentration, indicating that Bacteroidetes were tolerant to high levels of tetracycline $[1,11,12]$. Bacterial community structure and diversity have been reported using high-throughput sequencing. However, there is a lack of research on ARB community structure. Besides, this type of metagenomic sequencing data cannot provide direct evidence for ARB changes [7]. Even culturable bacteria also accounted for a low proportion of the total bacteria; the traditional pure culture method is the most direct method to understand ARB at present. Therefore, it is very meaningful that the development of antibiotic resistance of specific bacteria during composting should be monitored dynamically and analyzed comprehensively in combination with the traditional pure cultures.

Aerobic composting can effectively remove ARB from livestock manure. Previous studies concluded that cultured bacteria resistant to tetracycline and erythromycin decreased by 7 and 4 logs, respectively; substantial degradation of ampicillin and tetracycline-resistant Escherichia coli as well as a decrease in the abundance of erm and tet genes, was also observed during composting of cattle manure $[13,14]$. ARBs are the source and hosts for ARGs [4]. Most of the previous studies only analyzed the abundance of ARB and did not focus on the evolution of community structure. Besides, it is even little known that the influence of antibiotic types on the abundance and community structure of ARB, as well as the potential relationship of ARB and ARGs. In this study, chlortetracycline (CTC), sulfamethoxazole (SMX), ciprofloxacin (CIP), and LIN were selected according to their types and residual concentrations in our previous study [12]. These four antibiotics are frequently used in veterinary and human treatments worldwide, and have been detected in different environmental matrices [15]. The objectives of this research are to determine how (1) different antibiotics affect ARB abundance, (2) composting stage affects ARB communities, and (3) what relationships exist between ARB and ARGs to better mitigate public health risk. This study provides a better understanding on the fate of $A R B$ abundances and community structure, and ARGs in aerobic composting.

\section{Materials and methods Composting and sampling procedures}

The detailed experimental design was the same as in our previous article [12]. Briefly, the treatment without added antibiotic was used as the control (CK). Composting was performed in rectangular foam boxes $(55 \times 45 \times 45 \mathrm{~cm})$ with $2 \times 2 \mathrm{~cm}$ diameter holes spaced $15 \mathrm{~cm}$ apart in all 4 walls to allow facilitate ventilation. The basic physical and chemical properties of the composting materials are shown in Additional file 1: Table S1. Composting materials were composed of $14.28 \mathrm{~kg}$ pig manure:straw:coconut shell powder (6.67:4.94:2.67) with the C:N ratio of 30:1. Moisture content was set at $65 \%$. The residual concentrations of various antibiotics varied, but $50 \mathrm{mg} / \mathrm{kg}$ was selected as a standardized antibiotic concentration to ensure study comparability.

CTC, SMX, CIP and LIN obtained from Beijing DHLH Biotechnology Co. Ltd. China. CTC and LIN were mixed by methanol; SMX and CIP were dissolved in hydrochloric acid. Treatments were named as T0 (CK), T1 (CTC), T2 (SMX), T3 (LIN), T4 (CIP) and T5 (the four mixed antibiotics). All treatments were replicated for three times. The boxes were set in a row and ventilated for the duration of the 35-day experiment. Samples were collected on days $2,7,14,21,28$ and 35 , and at the top, middle and bottom of the reactor, then combined and mixed. The fresh samples were divided into two parts: one was stored at $4{ }^{\circ} \mathrm{C}$ for bacterial culture, and the other was stored in a $-80{ }^{\circ} \mathrm{C}$ refrigerator for the determination of ARGs. 


\section{Determination of the total culturable heterotrophic and antibiotic-resistant bacteria}

Samples $(5.00 \mathrm{~g})$ were added to a conical flask with $45 \mathrm{~mL}$ sterile water and then shaken at $200 \mathrm{rpm}$ and $25{ }^{\circ} \mathrm{C}$ for $30 \mathrm{~min}$. The total culturable heterotrophic bacteria (TCB) were determined by beef extract-peptone agar medium (Beijing Solarbio Science \& Technology Co. Ltd. China). In brief, the concentration of CTC, SMX and LIN and CIP in the different mediums was 16, 76,16 , and $4 \mu \mathrm{g} / \mathrm{mL}$, respectively, according to the Clinical and Laboratory Standards Institute guidelines to determine the abundance of ARB [16]. The plates were incubated for $24 \mathrm{~h}$ at $35{ }^{\circ} \mathrm{C}$. TCB and ARB levels were calculated from $\mathrm{CFU}$ on non-selective and selective mediums. Plates containing all four antibiotics were used to detect multiple antibiotic-resistant bacteria (MRB). ARBs were denoted as chlortetracycline-resistant bacteria (CRB), sulfamethoxazole-resistant bacteria (SRB), lincomycin-resistant bacteria (LRB), ciprofloxacin-resistant bacteria (CIRB), MRB, respectively.

\section{DNA extraction and quantitative polymerase chain reaction}

DNA extraction from samples was performed with a TIANamp Stool DNA Kit (Tiangen Biotech, Bejing, China) according to instructions provided by the manufacturer. DNA quality was checked on 1\% agarose gel. DNA concentration was determined by Nano Drop one (Thermo, USA). DNA samples stored at $-20{ }^{\circ} \mathrm{C}$ until use. Quantitative polymerase chain reaction (qPCR) was performed from the extracts to quantify tetracycline (tet $\mathrm{W}$, tet $\mathrm{X}$, tet $\mathrm{G}$, tet $\mathrm{O}$, and tet $\mathrm{A})$, sulfonamide (sul1 and sul2), macrolide $(\operatorname{erm} \mathrm{C}, \operatorname{erm} \mathrm{A}$, and $\ln u \mathrm{~A})$, quinolone-resistance genes (qnrA and qnrS), two integrons (int $\mathrm{I} 1$ and int $\mathrm{I} 2$ ), one multiple resistance gene (floR). The primers, sequence amplification length, and annealing temperature are detailed in Additional file 1: Table S2. The detailed protocols for qPCR are described in Additional file 1.

\section{Pretreatment of TCB and ARB, and sequence analysis}

Sequencing analysis was performed on single representative sample (days $0,7,21$, and 35 ) in different treatments. The pretreatment method referred to a previous study with some modifications [9]. All bacteria colonies in a plate were combined and washed with $\mathrm{ddH}_{2} \mathrm{O}$. Bacteria solution was mixed and centrifuged for $5 \mathrm{~min}(10,000 \mathrm{rpm})$ and then the supernatant was dumped. The $16 \mathrm{~S}$ rRNA sequence analysis was used to identify the taxonomic status of a bacterial species. The procedure used was as follows:
High throughput sequencing was conducted using the Illumina Miseq PE300 platform (Illumina, San Diego, CA, USA) by Shanghai Majorbio Bio-pharm Technology. The primers $338 \mathrm{~F}$ and $806 \mathrm{R}$ were used to target the V3-V4 region of $16 \mathrm{~S}$ rRNA gene [2]. The raw data was spliced and filtered for quality control and aggregated using Usearch software (http://www.drive5.com/ usearch/), and non-repeat sequences were identified and single sequences were removed to get rid of repetition. Clustering of operational taxonomic units (OTU) was conducted into minimum taxon using a similarity level of $97 \%$ to generate and a Biological Observation Matrix (BIOM) table. The Bayesian algorithm using the RDP (Ribosomal Database Project) classifier (https:// omictools.com/) was applied for the taxonomic analysis of representative OTU using Qiime (http://qiime.org/). This was accomplished by first extracting and added from each OTU as the representative sequence, compared with the RDB (Relational Database), and OTU was associated with bacterial species to form an OTU abundance table.

\section{Statistical analysis}

Basic statistical calculations were performed using Origin 9.1 (Origin Lab, San Diego, CA, USA). Significant differences were calculated using one-way analysis of variance (ANOVA) with IBM SPSS 23.0 Statistics (IBM, Chicago, ILL, USA). Heatmaps and principal co-ordinates analysis (PCoA) were constructed using Vegan, PCoA of $\mathrm{R}$ 3.5.2 (https://www.r-project.org/), respectively. The networks were built using Networkx software (http://netwo rkx.github.io/) according to the relative contents of each genus after classification.

\section{Results and discussion}

Variation in abundance of TCB and ARB at different stages of composting

Compared with Control (T0), the absolute abundance of TCB decreased in T1-T5, ranging from $1.48 \times 10^{14}$ to $3.04 \times 10^{11} \mathrm{CFU} / \mathrm{g}$ (Fig. 1). The addition of antibiotics affected on the bacterial community in the raw material. In $\mathrm{T} 5$, the absolute abundance of $\mathrm{TCB}$ was lower than other treatments. It was mainly because of the high concentration of antibiotics, which strongly affected microbial activity [17]. The absolute abundance of TCB decreased in the first 7 days of composting. The composting temperature was above $60{ }^{\circ} \mathrm{C}$, inhibiting the activity of some bacteria [12]. In addition, sudden changes in the living environment might inhibit the growth of various sensitive microbes [18]. The absolute abundance of TCB decreased by 4-5 logs in T3-T5 treatments, while only 2 logs in T1 and T2 treatments. This indicated that the abundance of TCB was also affected by antibiotic types. 


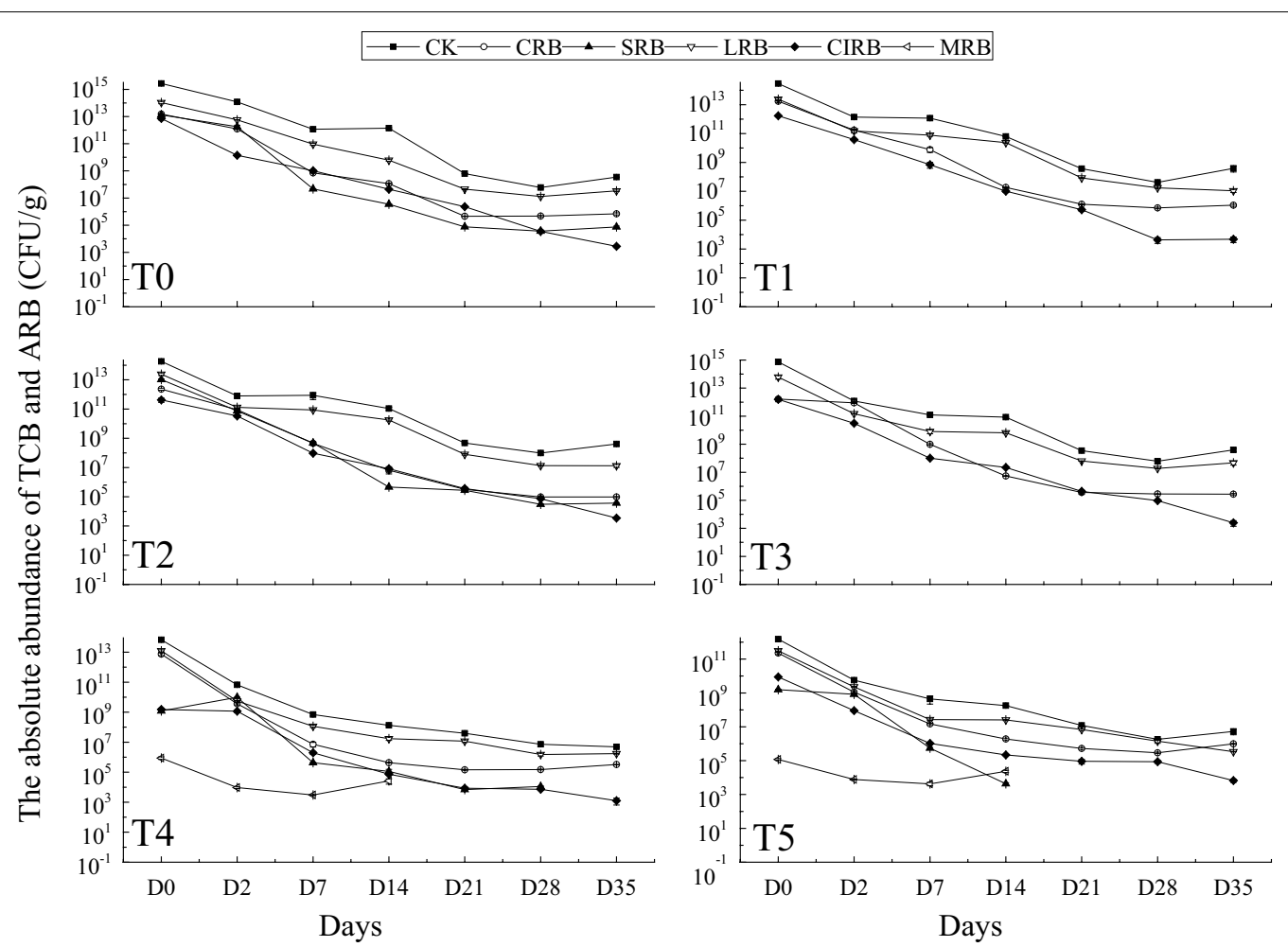

Fig. 1 Absolute abundance of total cultivable heterotrophic bacteria (TCB) and antibiotic-resistant bacteria (ARB). CRB chlortetracycline-resistant bacteria, SRB sulfamethoxazole-resistant bacteria, LRB lincomycin-resistant bacteria, CIRB ciprofloxacin-resistant bacteria, MRB multiple antibiotic-resistant bacteria. T0 is the control; $\mathrm{T} 1$ is chlortetracycline treatment; $\mathrm{T} 2$ is sulfamethoxazole treatment; $\mathrm{T} 3$ is lincomycin treatment; $\mathrm{T} 4$ is ciprofloxacin treatment; $T 5$ is multiple antibiotics treatment (The same below)

At the end of composting, the absolute abundance of TCB decreased to $6.96 \times 10^{7} \mathrm{CFU} / \mathrm{g}$ in T0; while, it was similar in the presence of CTC, SMX, and LIN (T1-T3). In contrast, TCB was reduced to $9.8 \times 10^{5} \mathrm{CFU} / \mathrm{g}$ in $\mathrm{T} 4$ and $1.06 \times 10^{6} \mathrm{CFU} / \mathrm{g}$ in T5 (Fig. 1). A previous study showed that most antibiotics were degraded rapidly within 13 days [19]. Selvam et al. (2012) also indicated that sulfadiazine was completely degradation within 3 days; however, CTC was within 21 days, $1.17 \mathrm{mg} / \mathrm{kg}$ of CIP was extracted after 56 days of composting [18]. The influence of CIP on bacterial community was more substantial than other antibiotics at the same concentration in composting. The most obvious influence was also observed on composting's physical and chemical properties in the treatment with CIP [12].

The populations of bacteria resistant to each antibiotic were not different from $\mathrm{T} 0$, with the abundance ranging from 21.6 to $1.44 \times 10^{12} \mathrm{CFU} / g$ (Fig. 1). In the first 2 days, the absolute abundance of ARB decreased gradually, while the relative abundance increased. For example, CRB increased from 5.90 to $12.85 \%$ in T1; SRB increased from 5.66 to $12.14 \%$ in T2 (Additional file 1: Fig. S1). The higher occurrence of ARB after composting was most likely due to mutation in common genes that thus extend their spectrum of resistance [20], transfer of ARGs among diverse bacteria (e.g., plasmids) [20], and selective pressure of the antibiotics [20,21]. In this study, mutation or horizontal transfer of CRB and SRB might be more likely to occur under the selection pressure of CTC or SMX. Therefore, the relative abundance of CRB and SRB increased within 2 days of composting. The absolute abundance of ARB was reduced by more than 6 orders of magnitude during the 35 days composting. On the one hand, ARB is also affected by temperature at the early stage of composting. On the other hand, the antibiotics degraded gradually over time. Yang et al. (2017) indicated that ARB was correlated with antibiotic concentrations [22]. Antibiotics degraded rapidly in the early stage of composting; thereby ARB did not increase at the end of composting. In $\mathrm{T} 4$ and $\mathrm{T} 5$, the absolute abundance of MRB was $0.24-1.75 \times 10^{4} \mathrm{CFU} / \mathrm{g}$ (Fig. 1). However, MRB was not found after day 21. It is possible that the combined action of high temperature and the four antibiotics inhibited the growth of MRB. Yang et al. (2016) have shown that Enterococcus was the most prevalent member of MRB (amoxicillin, kanamycin, gentamicin, 
and cephalexin) community (93.7\%) in the swine manure [23]. The results also showed that MRB was also affected by the type of antibiotics. No MRB was detected at the end of composting. The absolute abundance of four ARBs was $9.42 \times 10^{6}-2.51 \times 10^{2} \mathrm{CFU} / \mathrm{g}$, the order of ARB abundances was $\mathrm{LRB}>\mathrm{CRB}>\mathrm{SRB}>\mathrm{CIRB}>\mathrm{MRB}$ in the end (Fig. 1).

\section{Variation of TCB community structure}

Actinobacteria, Bacteroidetes, Firmicutes, and Proteobacteria were the primary phyla of TCB (Fig. 2). The abundance of Proteobacteria was $>65 \%$ in all treatments. The major genera were Escherichia and Shigella (Fig. 3). The abundance of Proteobacteria decreased, and the most apparent reduction was in T4, where they were reduced from 80 to $0.056 \%$ due to the abundance of Escherichia and Shigella decreased on day 7 (Fig. 3). Suhera et al. (2019) studies have shown that CIP had an inhibitory effect on almost all bacteria in the composting [24]. The combination of high temperature and CIP might be the reduction of culturable Proteobacteria. On days 7 and 21, Alcaligenes, Pseudomonas, Comamonas genus and Acinetobacter were main genera in T1, indicating a relative insensitivity or strong tolerance to CTC as compared with other antibiotics. On day 21, Comamonas genus displayed a notable decrease to $1 \%$, indicating that
Comamonas genus was sensitive to temperature and their decline correlated with CTC levels.

The abundance of Bacteroidetes was $20.36-0.65 \%$ in T0-T5, indicating that antibiotics impacted on Bacteroidetes in the initial stage. Sphingobacterium and Flavobacterium were dominant genera. On day 7 , Bacteroidetes increased to $36.59 \%, 29.24 \%$, and $34.76 \%$ in T0, T2, and $\mathrm{T} 5$, respectively, while decreased in other treatments (Fig. 2). Bacteroidetes has been shown to increase dramatically in cow manure and rice straw composts during the thermophilic phase due to the presence of thermophilic species [25]. This indicated that CTC, LIN and CIP inhibited the growth and reproduction of Bacteroidetes or even killed them. On day 21, Bacteroidetes increased to $29.80 \%$ and $25.72 \%$ due to the high abundance of Sphingobacterium in T4 and T5. In the end, Bacteroidetes decreased except for T2. This result was similar to Proteobacteria which was relatively heat-sensitive. The abundance of Bacteroidetes was increased, accounted for $62.02 \%$ in T2 due to Parapedobacter and Niabella rising to $36.61 \%$ and $21.51 \%$, respectively. In contrast, these genera were inhibited by SMX in the initial stage. They eventually regained growth or developed antibiotic resistance as the SMX was degraded and the temperature was reduced [3].

Actinobacteria was relatively low at $0.63-6.46 \%$ in all treatments. Actinobacteria increased to $61.09 \%$ and

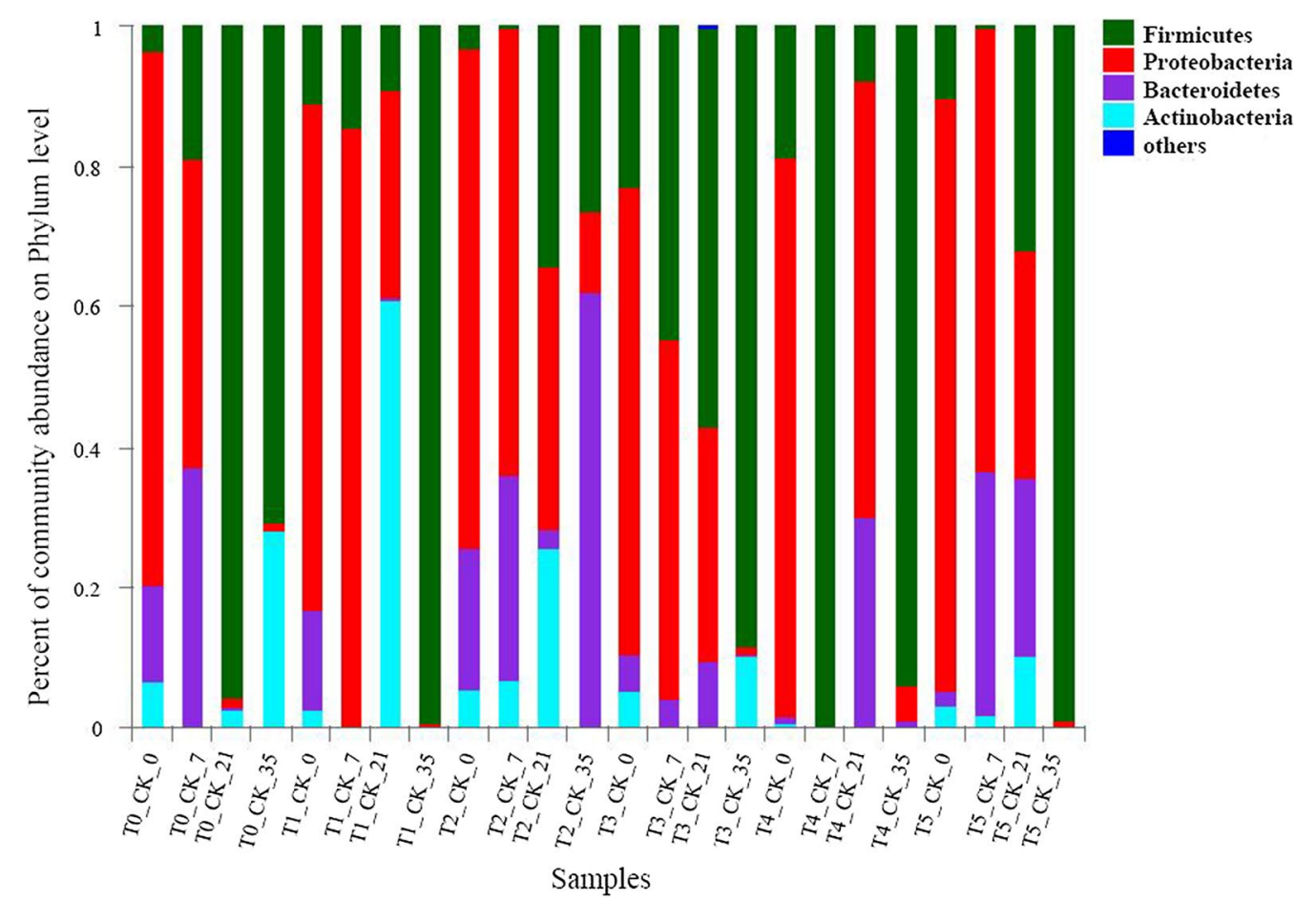

Fig. 2 Community abundance succession of total culturable heterotrophic bacteria in the phylum. 0, 7, 21, and 35 are the sampling time 


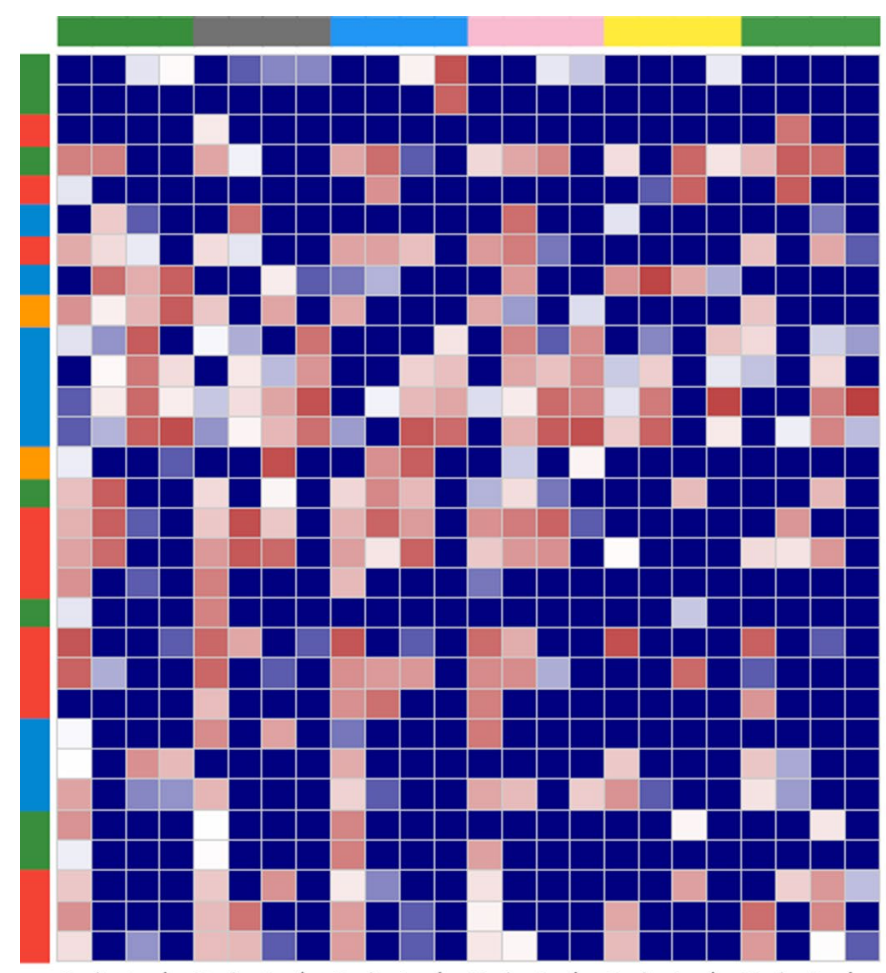

Parapedobacter

Niabella

Delftia

Sphingobacterium

Brevundimonas

Psychrobacillus

Acinetobacter

Solibacillus

Corynebacterium

Sporosarcina

unclassified_Planococcaceae

Bacillus

Lysinibacillus

Glutamicibacter

Flavobacterium

Comamonas

Pseudomonas

Citrobacter

unclassified_Sphingobacteriaceae

Escherichia and Shigella

Alcaligenes

Psychrobacter

Staphylococcus

Desemzia

Enterococcus

norank_Sphingobacteriaceae

Empedobacter

Stenotrophomonas

Enterobacter

unclassified_Enterobacteriaceae

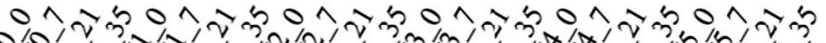

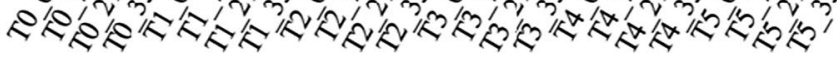

Fig. 3 Top 30 genera of total culturable heterotrophic bacteria

$25.67 \%$ due to the presence of Glutamicibacter spp. in T1 and T2 (Figs. 2, 3). Therefore, CTC and SMX inhibited the growth and reproduction of Actinobacteria, but Glutamicibacter spp. gradually recovered or either developed or was intrinsically resistant. In the end, only in T0 and T3, the abundance of Actinobacteria increased to $28 \%$ and $10.13 \%$, respectively (Fig. 2). In the absence of antibiotics, Corynebacterium was the dominant genus at $28 \%$ and demonstrated the resilience of this genus to composting, and has developed LIN resistance. These results were also consistent with a previous composting study which indicated Corynebacterium was a major genus [26].

The abundance of Firmicutes was $3.36-22.72 \%$ in all treatments (Fig. 2). The primary genera were Bacillus, Lysinibacillus, Sporosarcina, Enterococcus, and Staphylococcus (Fig. 3). Most genera were endospore formers and resistant to high temperatures [27]. Bacillus was isolated at the highest frequency in composting benefiting from their thermotolerance, and greatly impacted on degradation of waste $[28,29]$. The abundance of Firmicutes reached 99.94\%; the dominant genera were Lysinibacillus and Solibacillus in T4 (Figs. 2, 3). Both of these developed CIP resistance or a small resistant population became more abundant under CIP selection pressure. On day 35, Firmicute had a complete comparative advantage (except for T2), included Lysinibacillus, Bacillus, Solibacillus and Sporosarcina. The abundance of Firmicutes was relatively low because of the high proportion of Bacteroidetes in T2. These results indicated that the influence of antibiotics on microbial community structure was a continuous process despite the degradation of antibiotics.

\section{The community structure of ARB}

Compared with other phyla, Proteobacteria had the highest relative abundance in the initial stage, while Firmicutes became the dominant phylum with the extension of composting (Fig. 4). MRB was found only in the early stages of composting. On day 7, Proteobacteria (99.93\%) was the dominant MRB; Enterobacter was the main genus (Figs. 4, 5). Enterobacter is an opportunistic pathogenic genus that can cause extra-intestinal infections in the urinary, respiratory tracts, and wounds, which possess resistance to different antibiotics $[9,22]$. Alikkunju et al. (2016) study showed that the optimum growth temperature of Enterobacter was $25^{\circ} \mathrm{C}$ which exhibited enzyme production and good growth at a temperature range of $15-35^{\circ} \mathrm{C}$, pH $5-10$ [30]. In this study, the first 20 days of 

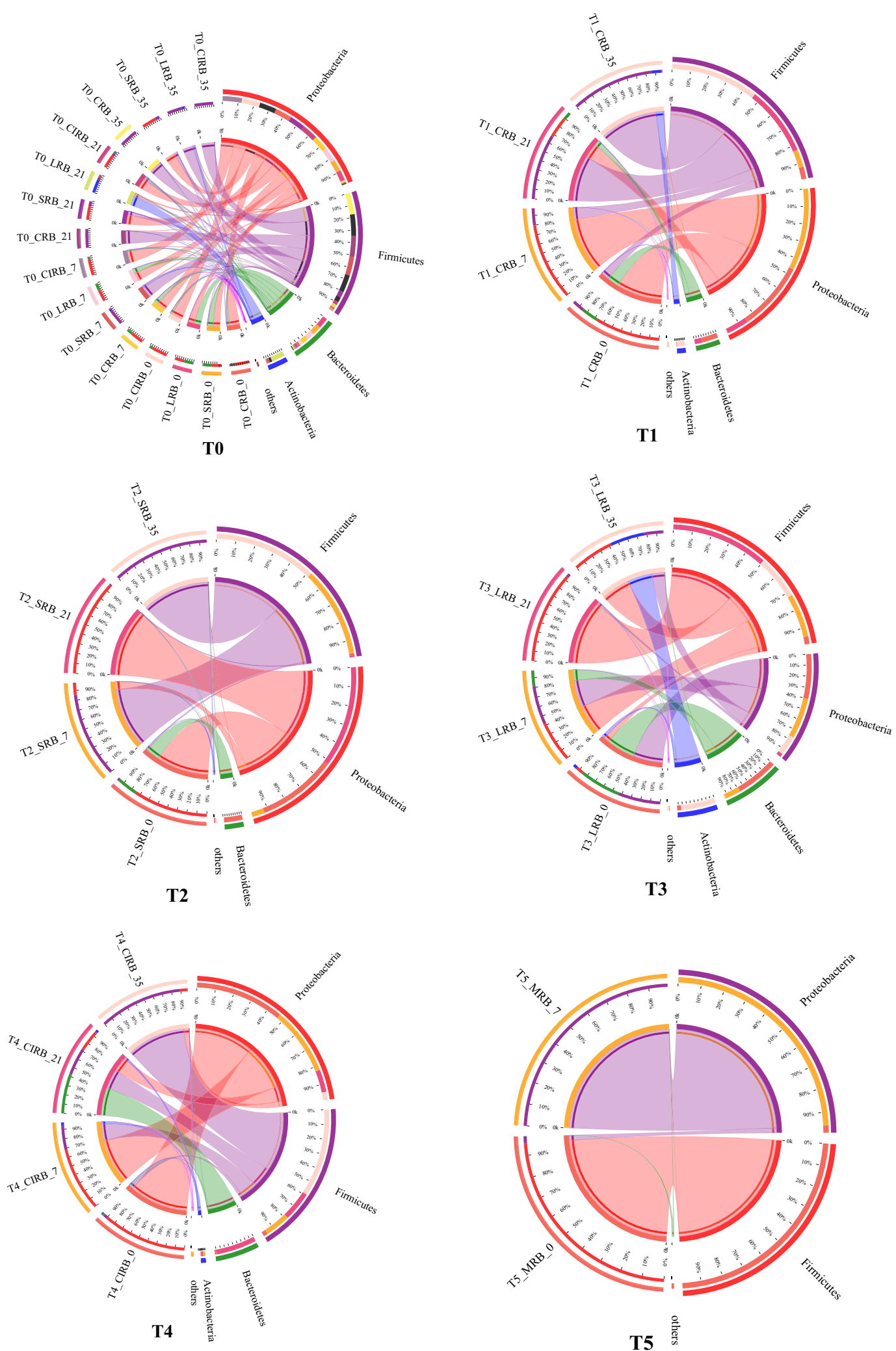

Fig. 4 Community abundance succession of antibiotic-resistant bacteria (ARB) in the phylum level 


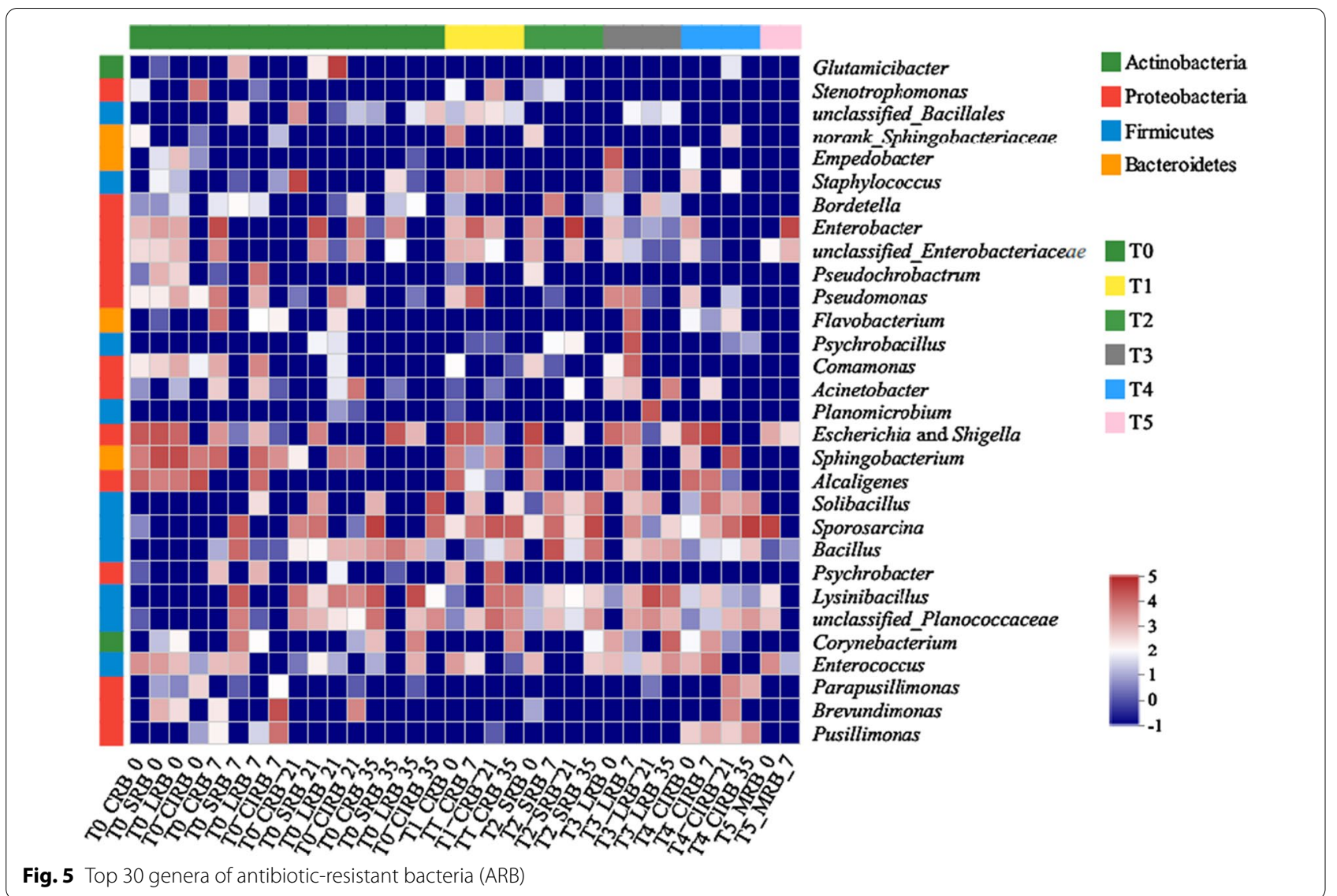

composting were above $50{ }^{\circ} \mathrm{C}$ [12]. Therefore, MRB was not detected at the end of composting.

The dominant CRB genera were Escherichia and Shigella, Enterobacter, Psychrobacter, Sphingobacterium, and Alcaligenes in the initial stage. Compared with Control (T0), the abundance of Escherichia and Shigella, and Pseudomonas increased, indicating that the existence of antibiotic enhanced bacterial resistance to CTC. As the composting continued, Firmicutes gradually replaced Proteobacteria becoming the primary CRB phylum. The dominant CRB genera were Sporosarcina, Lysinibacillus, Bacillus, Solibacillus. Corynebacterium accounted for $10.64 \%$ in T1. It is a facultative anaerobe and a pathogenic genus. Its CTC resistance was consistent with previous studies [31]. Composting generally had a heterogeneous population composed of animal and human pathogens that could cause disease in livestock [32, 33]. These findings suggested that CTC increased the risk of human pathogenic bacteria circulating in the environment and posed a threat to human health.

Proteobacteria and Bacteroidetes were the main SRB phyla in the initial stage (Fig. 4). The dominant genera included Escherichia and Shigella, Enterobacter, Alcaligenes, and Sphingobacterium (Fig. 5). The abundance of
Escherichia and Shigella increased in T2, indicating that SMX induced bacterial resistance, which was consistent with a previous study [34]. Escherichia and Shigella has been frequently reported in livestock and poultry feces, and was a common fecal-resistant microorganism [9, 35]. On day 7, Sporosarcina and Bacillus dominated at $23.79 \%$ and $54.49 \%$, respectively (Fig. 5). Liu et al. (2019) indicated that Bacillus could be associated with SMX removal [36]. These might explain the high abundance of Bacillus in the early stage. In the end, the dominant genera for SRB included Sporosarcina and Solibacillus in T2.

Proteobacteria and Bacteroidetes were also the main phyla of LRB in the early composting stage (Fig. 4). Escherichia and Shigella, Pseudomonas, and Empedobacter were the primary LRB in T3. Escherichia and Shigella, and Pseudomonas have been found to be resistant to various antibiotics $[9,22]$. Compared with T0, the abundance of Firmicutes increased, the dominant LRB were Psychrobacillus, Comamonas genus and Flavobacterium on day 7 , indicating that these genera showed strong tolerance to LIN. At the end of composting, Firmicutes, Actinobacteria and Proteobacteria dominated in the phyla of LRB with a ratio of $43.44 \%, 36.22 \%$ and $19.36 \%$, respectively (Fig. 4). In addition, Lysinibacillus, Acinetobacter and 
Corynebacterium were increased (Fig. 5). It was reported that Corynebacterium, a pathogenic genus, was a major genus in animal manure and organic fertilizer [32, 33]. It was also found to resistant to CTC. As a result, the risk of Corynebacterium should be considered in composting. Antibiotic residues in manure exerted a selective pressure on the bacterial community and induced the emergence of diverse ARGs even at low concentrations [37]. LIN might enhance horizontal transfer of ARGs, leading to the increase of LRB at phylum and genera, and indicating that the ecological risk of LIN was higher than other antibiotics.

CIP resistance was dramatically changed in T4 (Figs. 4, 5). The dominant CIRB was Proteobacteria in the first 21 days of composting; Firmicutes (98.87\%) became the dominant phylum in the end in T0. On day 7, Firmicutes increased, the dominant genera were Escherichia and Shigella, and Solibacillus (Figs. 4, 5). These genera were common ARB in manure. Previous researchers obtained similar results, who found the primary ARB was Enterobacter, Alcaligenes, Escherichia and Shigella, Acinetobacter, Enterococcus, and Bacillus in livestock and poultry manure [22]. In the end, Firmicutes was the absolute dominant phylum at $91.57 \%$, Sporosarcina was the dominant genus (Figs. 4, 5). A study monitored the bacterial community spiked with CIP at two different levels (2, $20 \mathrm{mg} / \mathrm{kg}$ ) during swine manure composting; found that Sporosarcina were selectively enriched in antibiotic treatments [18]. These results indicated that the sensitivity of Sporosarcina to CIP, and the potential selection of specific bacteria.

Previous studies have found that ARBs were Alcaligenes, Lampropedia, Escherichia, Enterococcus, Corynebacterium, Lactobacillus, Citrobacter, Bacillus and Pseudomonas [22]. Sporosarcina, Bacillus, Enterobacter, Lysinibacillus, Enterococcus, Escherichia and Shigella were resistant to all tested antibiotics in this study. The changes in the environment by the composting reduced the bacteria diversity, and favored the growth of tolerant bacteria [18]. This might be the reason for the difference between the results of ARB and those of previous studies. The widespread use of antibiotics has resulted in ARB development, which often co-carried aminoglycoside, quinolone, chloramphenicol, and sulfonamide resistance [38]. Bacteria developed antibiotic resistance due to the presence of ARGs; horizontal gene transfer was a primary reason driving alterations in antibiotic resistance. When ARB from feces entered the soil, they carried ARGs that were capable of horizontal transfer to indigenous bacteria and even to some human pathogens, such as Salmonella, Campylobacter, and Shigella $[37,39]$. In this study, ARB still existed at the end of composting. Therefore, ecological risks of manure needs to be evaluated.

\section{Analysis of differences in TCB and the relationship between ARB and ARGs}

Bacterial genera were reduced by 32 in $\mathrm{T} 4$ compared with other treatments (Additional file 1: Fig. S2). This indicated that CIP had the greatest impact on bacteria and reduced species richness, which was most likely the result of a wide bactericidal spectrum [24]. PCoA was used to identify any significant factors that influenced experimental results. PC1 and PC2 accounted for $75.92 \%$ of the changes in bacteria levels (Fig. 6). The bacterial community of different treatments was not separated, while there was a difference at different composting stages. These results indicated that alterations of the bacterial community structure in different treatments were primarily influenced by the different composting stages but not antibiotics types in composting. Previous studies concluded that the existence of antibiotics caused only a transient perturbation and the bacterial community structure was restored after 3 weeks of composting [18], and the microbial community could be affected by the composting materials and antibiotic concentration [40].

Based on the above results, an interactive network of 30 primary genera was generated to analyze the potential hosts of ARGs at different composting stages (Fig. 7). Only Psychrobacillus was significantly positively correlated with tetX $(p<0.05)$, indicating that Psychrobacillus might be the potential host of tetX on day 7. Psychrobacillus belongs to Firmicutes. A Previous study reported that Actinobacteria and Firmicutes were prevalent during the thermophilic phase of composting, and the prevalence of ARGs increased at the end of composting [41]. However, Solibacillus and Enterococcus of Firmicutes, Corynebacterium of Actinobacteria were significantly negatively correlated with various ARGs (Fig. 7a). In this study, the bacterial genera belonged to ARB. The culture-based methods captured only a small proportion of the whole bacterial community $(0.1-10 \%)$; ARB resistance cannot be mediated by the ARGs being analyzed [42]. Previous studies have shown that Bacteroidetes might be important hosts for tet $\mathrm{C}$, tet $\mathrm{X}$, sul2, and int I1 [43]. On day 7, Proteobacteria was the main phylum of ARB, and the abundance of ARB decreased (Fig. 4). These might be partially explaining the high degradation rate of ARGs (Additional file 1: Table S3). As the composting continued, we found that ermA was significantly positively correlated with Corynebacterium, Bhargavaea and unclassified-Bacillales, which might be the host bacteria or synergistic bacteria for ermA. A previous study indicated that the concentration of ARGs was positively correlated with the abundance of corresponding 


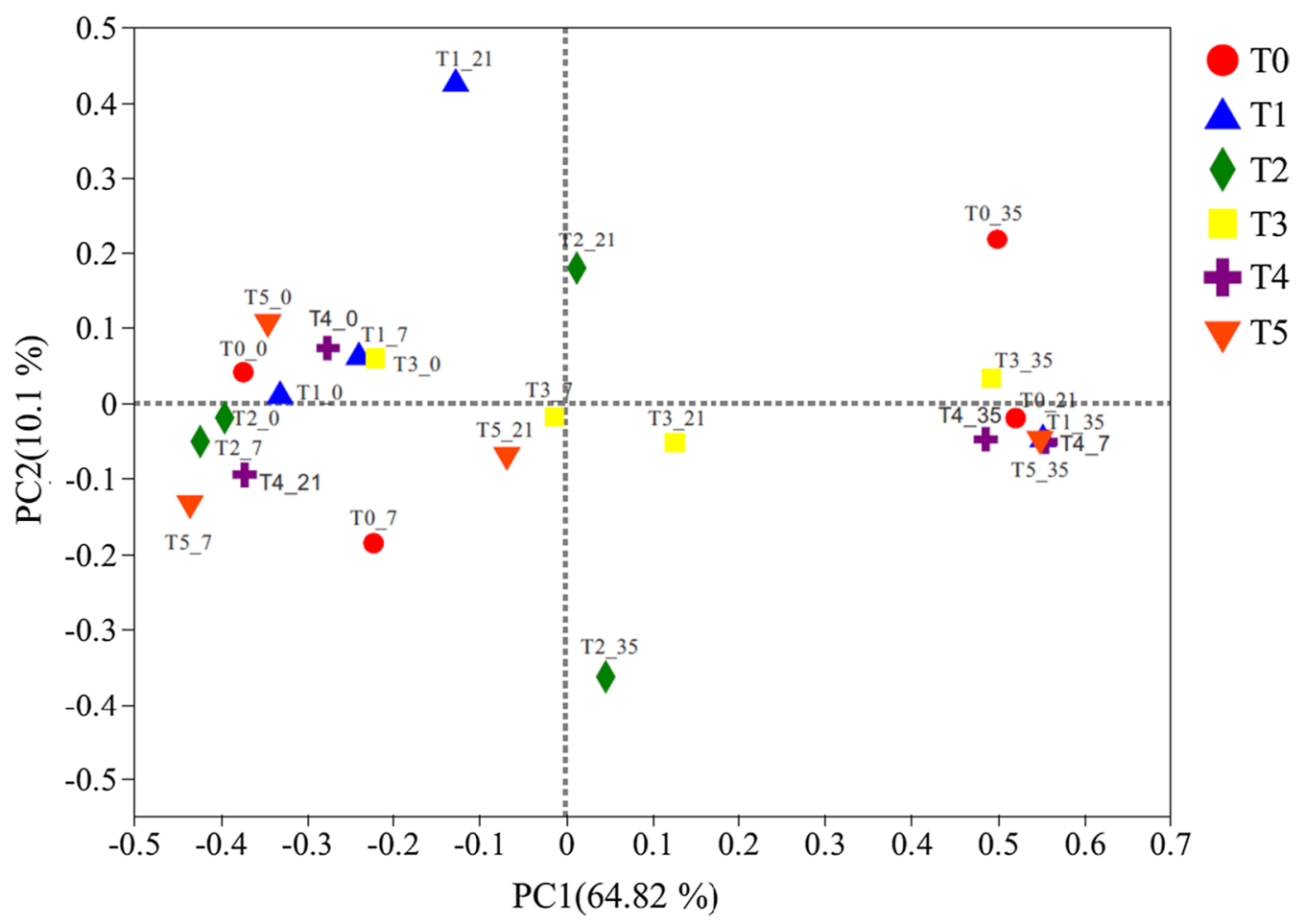

Fig. 6 Principal co-ordinates analysis (PCA) of total culturable heterotrophic bacteria (TCB)

bacteria hosts [44]. However, the degradation rate of ermA reached levels $>99 \%$ by 35 days (Additional file 1: Table S3). We found that ermA was correlated negatively with Solibacillus (Fig. 7c). The high degradation rate of ermA might be related to Solibacillus as the composting matured (Fig. 5). In addition, Escherichia and Shigella of Proteobacteria was reported as the carrier of tetA, Pseudomonas was the shelter of tet $\mathrm{A}$ and tet $\mathrm{G}$ [45]. Bacillus was significantly negatively correlated with both tetA and ermC (Fig. 7c). Similar results were found for tet $\mathrm{O}$. Overall, the decrease in abundance of culturable Proteobacteria and the increase of partially culturable Firmicutes (Solibacillus, Bacillus) might be explaining the high degradation rate of various ARGs with the progress of composting. Pathogenic bacteria (Corynebacterium, Acinetobacter, and Enterococcus) could not be completely removed, and the existence of antibiotics led to a partial proliferation (Fig. 5). Previous studies have shown that pathogenic bacteria were significantly correlated with ARGs, indicating that pathogenic bacteria could become a major host of ARGs [32, 46, 47]. Enterococcus was significantly positively correlated with tetX $(p<0.05)$. No significant positive correlations were observed between other pathogenic bacteria and ARGs. Anyhow, the spread risk of pathogenic bacteria to farmland soil and crops cannot be ignored in the application of swine manure.

\section{Conclusions}

In the first 7 days, the abundance of TCB decreased. At the end of composting, the lowest TCB abundance was found in $\mathrm{T} 4\left(9.8 \times 10^{5} \mathrm{CFU} / \mathrm{g}\right)$ and $\mathrm{T} 5$ $\left(1.06 \times 10^{6} \mathrm{CFU} / \mathrm{g}\right)$, respectively. Total ARB decreased, while their relative abundance increased on day 2 . CRB and SRB increased from 5.90 to $12.85 \%, 5.66$ to $12.14 \%$, respectively. LRB had the highest relative abundance in the whole composting process. The absolute abundance of $\mathrm{ARB}$ was $\mathrm{LRB}>\mathrm{CRB}>\mathrm{SRB}>\mathrm{CIRB}>\mathrm{MRB}$. Antibiotics led to a partial proliferation of ARBs including Corynebacterium, Sporosarcina, Solibacillus, and Acinetobacter. Especially Corynebacterium, a pathogenic genus, was observed in the treatments of CTC and LIN. In addition, the existence of LIN expanded the range of LRB at the phyla level (Firmicutes, Actinobacteria and Proteobacteria) at the end of composting. It indicated that these bacteria were more likely resistant to LIN, and thus the ecological risk of LIN was higher compared with other antibiotics studied in this work. PCA analysis showed that the composting stages primarily influenced the alteration of bacterial community structure. The network analysis indicated that the decrease in the abundance of culturable Proteobacteria and the increase of culturable Firmicutes might partially explain the high degradation rate of various ARGs 


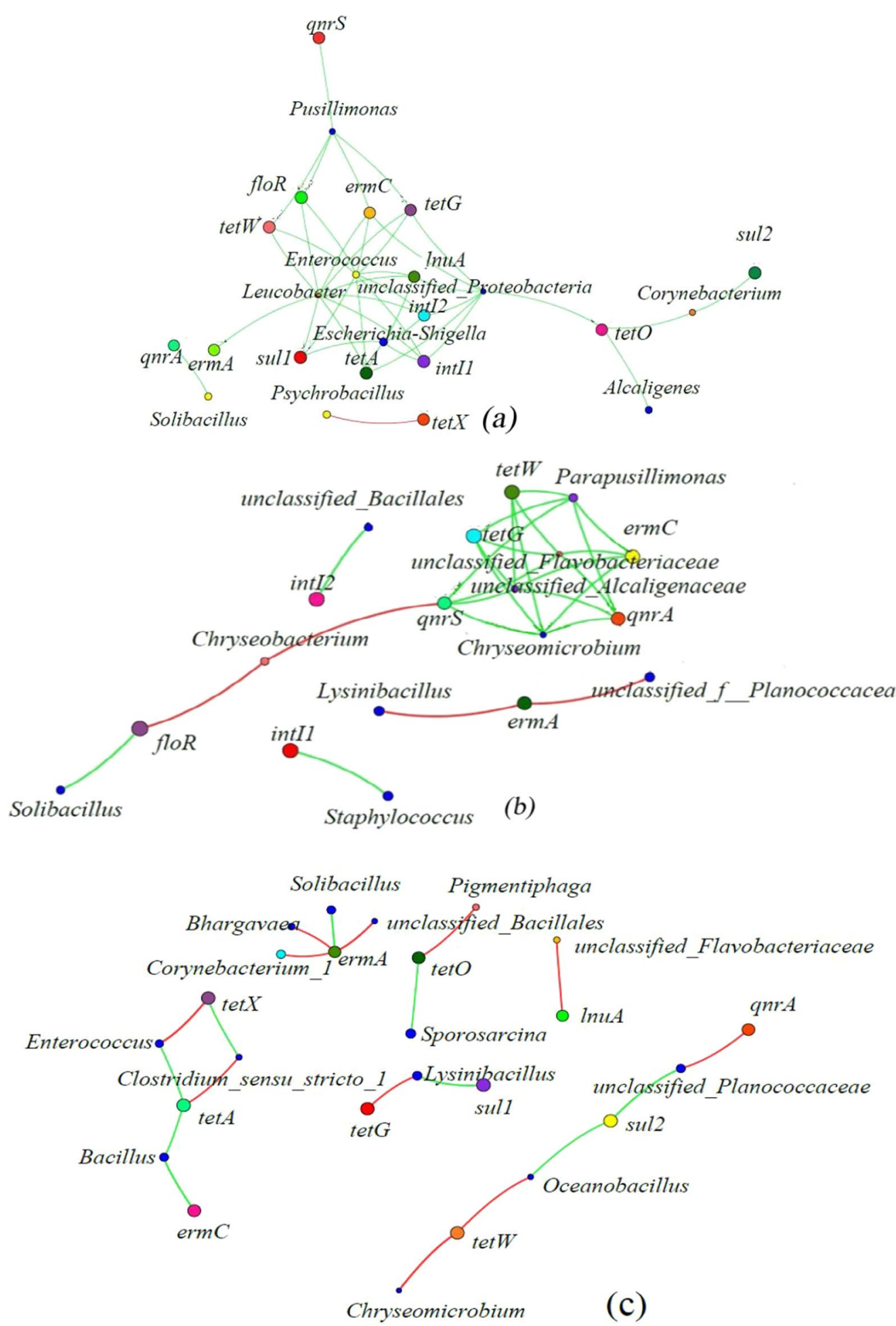

Fig. 7 Network analysis of ARB and ARGs. a day 7, $\mathbf{b}$ day 21, and $\mathbf{c}$ day 35. The calculated the Spearman rank correlation coefficient between species reflected the correlation between species. Genera with $p<0.05$ are shown by default. The node sizes represent genera abundance and different colors represent different genera at the phylum level. A red line color represents a positive correlation and green represents a negative correlation. Line thickness is proportional to the correlation coefficient 
in composting process. These results provide valuable reference for the ecological risk control of antibiotic resistance during swine manure composting.

\author{
Abbreviations \\ ARB: Antibiotic-resistant bacteria; ARGs: Antibiotic resistance genes; CFU: \\ Colony-forming units; CTC: Chlortetracycline; SMX: Sulfamethoxazole; CIP: \\ Ciprofloxacin; LIN: Lincomycin; TCB: Total culturable heterotrophic bacteria; \\ CRB: Chlortetracycline-resistant bacteria; SRB: Sulfamethoxazole-resistant bac- \\ teria; LRB: Lincomycin-resistant bacteria; CIRB: Ciprofloxacin-resistant bacteria; \\ MRB: Multiple antibiotic-resistant bacteria; QPCR: Quantitative polymerase \\ chain reaction; OUT: Operational taxonomic units; BIOM: Biological observa- \\ tion matrix; RDP: Ribosomal database project; RDB: Relational database; PCoA: \\ Principal co-ordinates analysis.
}

\section{Supplementary Information}

The online version contains supplementary material available at https://doi. org/10.1186/s12302-021-00535-6.

Additional file 1: Fig. S1. The relative abundance of ARB to TCB during composting. ARB is antibiotic-resistant bacterial; TCB is total culturable heterotrophic bacteria. T0 is the control; $\mathrm{T} 1$ is chlortetracycline treatment; $\mathrm{T} 2$ is sulfamethoxazole treatment; $\mathrm{T} 3$ is lincomycin treatment; T4 is ciprofloxacin treatment; T5 is multiple antibiotics treatment. CRB: chlortetracycline-resistant bacteria; SRB: sulfamethoxazole-resistant bacteria; LRB: lincomycin-resistant bacteria; CIRB: ciprofloxacin-resistant bacteria; MRB: multiple antibiotic-resistant bacteria. Fig. S2. TCB present in the composting process. The bar chart indicates the total number of species in each group at the selected taxonomic level. Lower; abscissa indicates the number of common or unique groups, and the length of the horizontal column above represents the corresponding number of species. Table S1. Physical and chemical properties of raw materials used for composting. Table S2. aPCR primers used in this study. Table S3. The degradation rate of ARGs in different stage of the swine manure composting (days $7,21,35)$.

\section{Acknowledgements}

\section{Not applicable.}

\section{Authors' contributions}

TS and $\mathrm{HL}$ contributed to the experiments, original draft writing and revising. $\mathrm{BL}, J Y, M S$ and $M Y$ were responsible for the data analysis. $L L$ and $Y T$ were contributors in the data visualization. SX and CZ were responsible for manuscript correction and supervision. All authors read and approved the manuscript.

\section{Funding}

This research was funded by the National Water Pollution Control and Treatment Science and Technology Major Project in China (2017ZX07401002), the Natural Science Foundation of Beijing, China (6192029), the Young Elite Scientist Sponsorship Program by CAST (YESS) (2018QNRC001).

\section{Availability of data and materials}

The datasets obtained and analyzed during the current study are available from the corresponding author upon reasonable request.

\section{Declarations}

Ethics approval and consent to participate

Not applicable.

\section{Consent for publication}

Not applicable.

\section{Competing interests}

The authors declared that they have no known competing personal relationships or financial interests that could have appeared to affect the work reported in this paper.

\section{Author details}

${ }^{1}$ Institute of Environment and Sustainable Development in Agriculture, Chinese Academy of Agricultural Sciences, Beijing 100081, People's Republic of China. ${ }^{2}$ College of Forestry, Northwest A\&F University, Yangling, Shaanxi 712100, People's Republic of China. ${ }^{3}$ State Key Laboratory of Soil Erosion and Dryland Farming on the Loess Plateau, Institute of Soil and Water Conservation, Northwest A\&F University, Yangling, Shaanxi 712100, People's Republic of China.

Received: 14 April 2021 Accepted: 31 July 2021

Published online: 11 August 2021

\section{References}

1. Chen Z, Wang Y, Wen Q (2018) Effects of chlortetracycline on the fate of multi-antibiotic resistance genes and the microbial community during swine manure composting. Environ Pollut 237:977-987

2. Ren S, Lu A, Guo X, Zhang Q, Wang Y, Guo X, Wang L, Zhang B (2019) Effects of co-composting of lincomycin mycelia dregs with furfural slag on lincomycin degradation, degradation products, antibiotic resistance genes and bacterial community. Bioresour Technol 272:83-91

3. Sardar MF, Zhu CX, Geng B, Huang YL, Abbasi B, Zhang ZG, Song TT, Li HN (2021) Enhanced control of sulfonamide resistance genes and host bacteria during thermophilic aerobic composting of cow manure. Environ Pollut 275:116587

4. Liu N, Xu L, Han L, Huang G, Ciric L (2020) Microbiological safety and antibiotic resistance risks at a sustainable farm under large-scale open-air composting and composting toilet systems. J Hazard Mater 401:123391

5. Staley ZR, Schmidt AM, Woodbury B, Eskridge KM, Durso L, Li X (2020) Corn stalk residue may add antibiotic-resistant bacteria to manure composting piles. J Environ Qual 49(3):745-753

6. Lewis WH, Tahon G, Geesink P, Sousa DZ, Ettema TJG (2020) Innovations to culturing the uncultured microbial majority. Nat Rev Microbiol 19:225-240

7. Bengtsson-Palme J, Hammaren R, Pal C, Ostman M, Bjorlenius B, Flach CF, Fick J, Kristiansson E, Tysklind M, Larsson DGJ (2016) Elucidating selection processes for antibiotic resistance in sewage treatment plants using metagenomics. Sci Total Environ 572:697-712

8. Brooks JP, Adeli A, Mclaughlin MR (2014) Microbial ecology, bacterial pathogens, and antibiotic resistant genes in swine manure wastewater as influenced by three swine management systems. Water Res 57:96-103

9. Yang Q, Ren S, Niu T, Guo Y, Qi S, Han X, Liu D, Pan F (2014) Distribution of antibiotic-resistant bacteria in chicken manure and manure-fertilized vegetables. Environ Sci Pollut Res 21(2):1231-1241

10. Chen J, Michel FC, Sreevatsan S, Morrison M, Yu Z (2010) Occurrence and persistence of erythromycin resistance genes (erm) and tetracycline resistance genes (tet) in waste treatment systems on swine farms. Microb Ecol 60(3):479-486

11. Lv B, Xing M, Yang J (2018) Exploring the effects of earthworms on bacterial profiles during vermicomposting process of sewage sludge and cattle dung with high-throughput sequencing. Environ Sci Pollut Res 25(13):12528-12537

12. Song TT, Zhu CX, Xue S, Li BX, Ye J, Geng B, Li LF, Sardar FM, Li N, Feng S, Li HN (2020) Comparative effects of different antibiotics on antibiotic resistance during swine manure composting. Bioresour Technol 315:123820

13. Sharma R, Larney FJ, Chen J, Yanke LJ, Morrison M, Topp E, Mcallister TA, Yu Z (2009) Selected antimicrobial resistance during composting of manure from cattle administered sub-therapeutic antimicrobials. J Environ Qual 38(2):567-575

14. Wang L, Gutek A, Grewal S, Michel FC, Yu Z (2015) Changes in diversity of cultured bacteria resistant to erythromycin and tetracycline in swine manure during simulated composting and lagoon storage. Lett Appl Microbiol 61(3):245-251

15. Pan M, Chu LM (2017) Fate of antibiotics in soil and their uptake by edible crops. Sci Total Environ 599-600:500-512 
16. Clinical and Laboratory Standards Institute (CLSI) (2010) Performance standards for antimicrobial susceptibility testing; twentieth informational supplement, vol 30. Clinical and Laboratory Standards Institute

17. Yang CW, Hsiao WC, Chang BV (2016) Biodegradation of sulfonamide antibiotics in sludge. Chemosphere 150:559-565

18. Selvam A, Xu DL, Zhao ZY, Wong JWC (2012) Fate of tetracycline, sulfonamide and fluoroquinolone resistance genes and the changes in bacterial diversity during composting of swine manure. Bioresour Technol 126:383-390

19. Ho YB, Zakaria MP, Latif PA, Saari N (2013) Degradation of veterinary antibiotics and hormone during broiler manure composting. Bioresour Technol 131:476-484

20. Costanzo SD, Murby J, Bates J (2005) Ecosystem response to antibiotics entering the aquatic environment. Mar Pollut Bull 51(1-4):218-223

21. Martinez JL (2009) Environmental pollution by antibiotics and by antibiotic resistance determinants. Environ Pollut 157(11):2893-2902

22. Yang Q, Tian T, Niu T, Wang P (2017) Molecular characterization of antibiotic resistance in cultivable multidrug-resistant bacteria from livestock manure. Environ Pollut 229:188-198

23. Yang QX, Wang RF, Ren SW, Szoboszlay M, Moe LA (2016) Practical survey on antibiotic-resistant bacterial communities in livestock manure and manure-amended soil. J Environ Sci Health Part B 51(1):14-23

24. Suhera MA, Bassma MD, Ebtisam AA (2019) Effect of ciprofloxacin on S. aureus and $E$. coli growth in presence of vitamin C using cup cut diffusion method. J Pharm Pharm 8:473-484

25. Albuquerque L, Polonia ARM, Barroso C, Froufe HJC, Lage O, Lobo-DaCunha A, Egas C, Da Costa MS (2018) Raineya orbicola gen. nov., sp. Nov. a slightly thermophilic bacterium of the phylum Bacteroidetes and the description of Raineyaceae fam. Nov. Int. J Syst Evol Microbiol 68(4):982-989

26. Li X, Shi XS, Lu MY, Zhao YZ, Li X, Peng H, Guo RB (2019) Succession of the bacterial community and functional characteristics during continuous thermophilic composting of dairy manure amended with recycled ceramsite. Bioresour Technol 294:122044

27. Haakensen M, Dobson CM, Deneer H, Ziola B (2008) Real-time PCR detection of bacteria belonging to the Firmicutes Phylum. Int J Food Microbiol 125(3):236-241

28. De Gannes V, Eudoxie G, Hickey WJ (2013) Prokaryotic successions and diversity in composts as revealed by 454-pyrosequencing. Bioresour Technol 133:573-580

29. Gao M, Yang Z, Guo Y, Chen M, Qiu T, Sun X, Wang X (2020) The size distribution of airborne bacteria and human pathogenic bacteria in a commercial composting plant. Front Environ Sci Eng 15(3):39

30. Alikkunju AP, Sainjan N, Silvester R, Joseph A, Rahiman M, Antony AC, Kumaran RC, Hatha M (2016) Screening and characterization of cold-Active $\beta$-Galactosidase producing psychrotrophic Enterobacter ludwigii from the sediments of Arctic Fjord. Appl Biochem Biotechnol 180:477-490

31. Ghosh S, Lapara TM (2007) The effects of subtherapeutic antibiotic use in farm animals on the proliferation and persistence of antibiotic resistance among soil bacteria. ISME J 1(3):191-203

32. Xu Y, Li HY, Shi RG, Lv JP, Li BH, Yang FX, Zheng XQ, Xu J (2020) Antibiotic resistance genes in different animal manures and their derived organic fertilizer. Environ Sci Eur 32:102

33. Mohammadi NS, Mafakheri S, Abdali N, Barcena-Uribarri I, Tauch A, Benz R (2013) Identification and characterization of the channel-forming protein in the cell wall of Corynebacterium amycolatum. BBA-Biomembranes 1828:2574-2582
34. Graves AK, Liwimbi L, Israel DW, Van Heugten E, Robinson B, Cahoon CW, Lubbers JF (2011) Distribution of ten antibiotic resistance genes in E. coli isolates from swine manure, lagoon effluent and soil collected from a lagoon waste application field. Folia Microbiol (Praha) 56(2):131-137

35. Jiang HX, Lu DH, Chen ZL, Wang XM, Chen JR, Liu YH, Liao XP, Liu JH, Zeng ZL (2011) High prevalence and widespread distribution of multi-resistant Escherichia coli isolates in pigs and poultry in China. Vet J 187(1):99-103

36. Liu Q, Li M, Liu R, Zhang Q, Wu D, Zhu D, Shen X, Feng C, Zhang F, Liu X (2019) Removal of trimethoprim and sulfamethoxazole in artificial composite soil treatment systems and diversity of microbial communities. Front Environ Sci Eng. https://doi.org/10.1007/s11783-019-1112-9

37. Fang H, Wang HF, Cai L, Yu YL (2014) Prevalence of antibiotic resistance genes and bacterial pathogens in long term manured greenhouse soils as revealed by metagenomic survey. Environ Sci Technol 16:1095-1104

38. Philippon A, Arlet G, Jacoby GA (2002) Plasmid-determined AmpC-type beta-lactamases. Antimicrob Agents Chemother 46(1):1-11

39. Xie YF, Li XW, Wang JF, Christakos G, Hu MG, An LH, Li FS (2012) Spatial estimation of antibiotic residues in surface soils in a typical intensive vegetable cultivation area in China. Sci Total Environ 430:126-131

40. Jindo K, Sanchez-Monedero MA, Hernandez T, Garcia C, Furukawa T, Matsumoto K, Sonoki T, Bastida F (2012) Biochar influences the microbial community structure during manure composting with agricultural wastes. Sci Total Environ 416:476-481

41. Guo H, Gu J, Wang X, Yu J, Nasir M, Peng H, Zhang R, Hu T, Wang Q, Ma J (2019) Responses of antibiotic and heavy metal resistance genes to bamboo charcoal and bamboo vinegar during aerobic composting. Environ Pollut 252:1097-1105

42. Littlefield-wyer JG, Brooks P, Katouli M (2008) Application of biochemical fingerprinting and fatty acid methyl ester profiling to assess the effect of the pesticide atradex on aquatic microbial communities. Environ Pollut 153:393-400

43. Rampelli S, Schnorr SL, Consolandi C, Turroni S, Severgnini M, Peano C, Brigidi P, Crittenden AN, Henry AG, Candela M (2015) Metagenome sequencing of the hadza hunter-gatherer gut microbiota. Curr Biol 25(13):1682-1693

44. Li B, Zhang XX, Guo F, Wu WM, Zhang T (2013) Characterization of tetracycline resistant bacterial community in saline activated sludge using batch stress incubation with high-throughput sequencing analysis. Water Res 47(13):4207-4216

45. Chopra I, Roberts M (2001) Tetracycline antibiotics: mode of action, applications, molecular biology, and epidemiology of bacterial resistance. Mol Biol Rev 65(2):232-260

46. Lu LH, Zeng G, Fan CZ, Zhang JC, Chen AW, Chen M, Jiang M, Yuan YJ, Wu HP, Lai MY, He YB (2014) Diversity of Two-domain laccase-like multicopper oxidase genes in Streptomyces spp.: identification of genes potentially involved in extracellular activities and lignocellulose degradation during composting of agricultural waste. Appl Environ Microbiol 80:3305-3314

47. Wang $H X$, Wang $N$, Wang $B$, Zhao Q, Fang H, Fu CW, Tang CX, Jiang F, Zhou Y, Chen Y, Jiang QW (2016) Antibiotics in drinking water in shanghai and their contribution to antibiotic exposure of school children. Environ Sci Technol 50:2692-2699

\section{Publisher's Note}

Springer Nature remains neutral with regard to jurisdictional claims in published maps and institutional affiliations. 